\title{
The role of plasmapheresis in organophosphate poisoning: Case reports of three pediatric patients
}

\author{
Ufuk Yükselmiş ${ }^{1}$, Mustafa Özçetin², Yakup Çağ ${ }^{1}$, Dinçer Yıldızdaş ${ }^{3}$, Hayri Levent Yılmaz \\ ${ }^{1}$ Department of Pediatrics, Dr. Lutfi Kirdar Kartal Training and Research Hospital, University of Health Sciences, Istanbul; \\ ${ }^{2}$ Department of Pediatrics, Istanbul University Istanbul Faculty of Medicine, Istanbul; Departments of ${ }^{3}$ Pediatric Intensive \\ Care, ${ }^{4}$ Pediatric Emergency, Cukurova University Faculty of Medicine, Adana, Turkey. E-mail: mozcetin@gmail.com \\ Received: 12th December 2016, Revised: 26th February 2017, Accepted: 28th February 2017
}

SUMMARY: Yükselmiş U, Özçetin M, Çağ Y, Yıldızdaş D, Yılmaz HL. The role of plasmapheresis in organophosphate poisoning: Case reports of three pediatric patients. Turk J Pediatr 2017; 59: 491-496.

The aim of the study was to assess the impact of plasmapheresis treatment in the management of three pediatric patients with organophosphate poisoning who did not respond to standard treatment. The treatment of signs and symptoms, and supportive treatment has been evaluated in this paper.

Patients were initially given atropine infusion and $0.05 \mathrm{mg} / \mathrm{kg}$ atropine with five-minute intervals. Despite pralidoxime loading and three consecutively infusions clinical symptoms did not respond to the treatment and plasma pseudocholinesterase levels did not decrease. At this point, plasmapheresis was used on three consecutive days. Accordingly, the clinical signs improved and mechanical ventilation was no longer necessary. Patients were discharged on the sixteenth day after their admission to the hospital.

Plasmapheresis may be an option for the patients who do not respond to atropine and pralidoxime treatment in organophosphate poisoning.

Key words: organophosphate, poisoning, plasmapheresis.

Organophosphates (OP) are toxic chemicals that are found in most pesticides. Therefore, OP poisoning is quite common in developing countries. ${ }^{1}$ These compounds are absorbed by skin, gastrointestinal system and respiratory system. Since absorption by skin is slower and symptoms occur more slowly, patients are usually late for hospital admittance hence skin exposures may have more serious results. ${ }^{2}$

Compounds are classified according to the atoms on the phosphate. Compounds are named as phosphates when their four atoms contain oxygen; as phosphothioates when containing sulfur; as phosphoramides when containing nitrogen; as phoshoramidothionates when containing nitrogen and sulfur; as phosphonates when containing carbon and as phosphothionates when containing carbon and sulfur. Elimination of phophothioates; because of their lipophilic composition, is slower than phosphates. OP compounds are mainly metabolized in liver by hydrolysis and show variations between compounds.
Compounds like methylparation and diazinon are highly liposoluble and may cause late toxicity symptoms because of re-release in fat tissue. Compounds are required to be in oxone forms for inhibition. Thioates which are not accepted as important inhibitors, may be activated by metabolisation with oxones. The cleansing rate of the oxons vary according to the characteristics of the compound. ${ }^{2}$

OP's cause accumulation of acetylcholine (ACh) in the synapses by inhibiting the enzyme acethylcholinesterase (AChE). Since diethyl organophosphates are inactivated more slowly than dimethyl organophosphates, the reactivation of the enzyme AChE is slower. ${ }^{3}$ The traditional approach which is to treat OP poisoning with atropine and oximes, has been shown to be insufficient to prevent morbidity and mortality in former studies. ${ }^{3-5}$

The purpose of plasmapheresis is to separate antibodies, immunocomplexes, endogenous and exogenous toxins from the plasma and 
replace them with some plasma proteins and coagulation factors. Plasmapheresis, colloids, fresh frozen plasma and albumin are used for this purpose. Unlike albumin, fresh frozen plasma contains active cholinesterase, and it is used for increasing the cholinesterase level of patients with low plasma cholinesterase levels. 6,7

In this paper, we aimed to present three patients who recovered with plasmapheresis after OP poisoning.

\section{Case 1}

A 7-year-old female patient, with vomiting, abdominal pain and progressive deterioration of consciousness, was brought to our pediatric emergency service (ES) 3 days after her hair was washed with an agricultural pesticide containing OP for head lice. At admittance, her body temperature was $37^{\circ} \mathrm{C}$, respiratory rate was $25 / \mathrm{min}$, blood pressure was $120 / 80$ $\mathrm{mmHg}$, heart rate was $161 / \mathrm{min}$, and $\mathrm{SpO} 2$ was $95 \%$. Her general condition was poor. She was intubated with miotic and pinpoint pupils, rough respiratory sounds and widespread crepitane rales. She had thick secretion in oropharynx and hypopharynx, GCS (Glascow Coma Scale) was 10/15. Plasma pseudocholinesterase level was $428 \mathrm{U} / \mathrm{L}$ (normal range: 3,530-10,800 U/L). The patient was diagnosed with OP poisoning based on clinical and laboratory findings.
Since she had a history of exposure to OP by her hair being washed with an agricultural chemical, her hair was cut and all of her body and the remaining hair were cleansed with soap and water. Monitoring clinical signs, she was given atropine at the dose of $0.05 \mathrm{mg} / \mathrm{kg} /$ hour every 5 minutes. Since heavy bronchorrhea and secretions continued despite intermittent atropine infusion, atropine infusion dose was augmented to $0.08 \mathrm{mg} / \mathrm{kg} /$ hour and injection of pralidoxime (PAM) at $40 \mathrm{mg} / \mathrm{kg} /$ dose was applied, followed by PAM infusion $(8 \mathrm{mg} / \mathrm{kg}$ / hour) for two days. In spite of sixty hours of intensive therapy, the clinical findings did not improve and plasmapheresis was planned. The patient received plasmapheresis in three consecutive days. On the fourth day, the clinical findings improved and the pseudocholinesterase level of $3922 \mathrm{U} / \mathrm{L}$ rose up to $4868 \mathrm{U} / \mathrm{L}$ (Fig. 1). Atropine was infused every 20 minutes after the first plasmapheresis, every 30 minutes after the second plasmapheresis, and every hour after the third plasmapheresis. The patient was separated from mechanical ventilation and she gained consciousness. Atropine dose was decreased gradually and terminated on the twelfth day. The patient was discharged on the sixteenth day.

\section{Case 2}

A 5-year-old female patient who was the sibling

Table I. Plasma Treatment and Techiques Used in Cases.

\begin{tabular}{|c|c|c|c|}
\hline & Case-1 & Case-2 & Case-3 \\
\hline Weight (kg) & 20 & 18 & 42 \\
\hline Hematocrit (\%) & 39 & 34 & 41 \\
\hline Total blood volume & $1,400 \mathrm{ml}$ & $1,260 \mathrm{ml}$ & $2,520 \mathrm{ml}$ \\
\hline $\begin{array}{l}\text { Technique of the plasma } \\
\text { exchange }\end{array}$ & $\begin{array}{c}\text { Centrifugation technique } \\
\text { (Spectra Optica; } \\
\text { CardianBCT, Lakewood, } \\
\text { CO, USA, and Com.Tec; } \\
\text { Fresenius HemoCare } \\
\text { GmbH, Bad Homburg, } \\
\text { Germany) }\end{array}$ & $\begin{array}{c}\text { Centrifugation } \\
\text { technique (Spectra } \\
\text { Optica; CardianBCT, } \\
\text { Lakewood, CO, USA, } \\
\text { and Com.Tec; Fresenius } \\
\text { HemoCare GmbH, Bad } \\
\text { Homburg, Germany) }\end{array}$ & $\begin{array}{l}\text { Continuous } \\
\text { filtration technique } \\
\text { (Prisma; Gambro, } \\
\text { Lund, Sweden) }\end{array}$ \\
\hline $\begin{array}{l}\text { TPV given on the first } \\
\text { day }\end{array}$ & $1281 \mathrm{ml}$ & $1247 \mathrm{ml}$ & $1486 \mathrm{ml}$ \\
\hline $\begin{array}{l}\text { TPV given on the other } \\
\text { days }\end{array}$ & $854 \mathrm{ml}$ & $831 \mathrm{ml}$ & $1486 \mathrm{ml}$ \\
\hline Catheter type & Femoral & Femoral & Femoral \\
\hline Plasmapheresis duration & 3 days & 3 days & 3 days \\
\hline
\end{tabular}

TPV: total plasma volume [total blood volume $\mathrm{x}$ (1-hematocrit)] 
Case 1

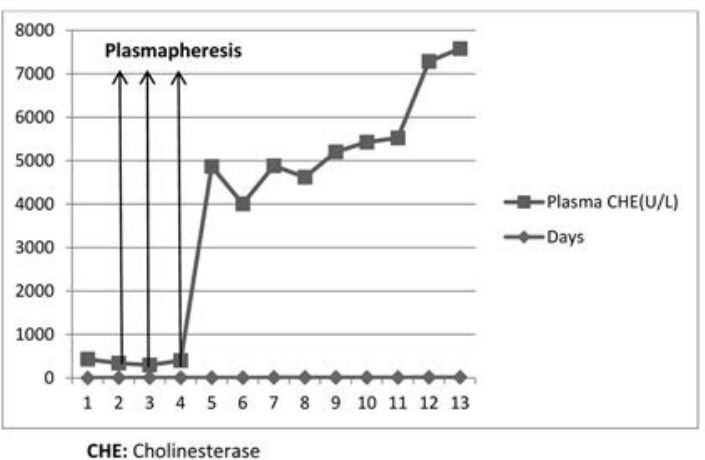

Fig. 1. The level of cholinesterase before and after plasmapheresis in Case 1.

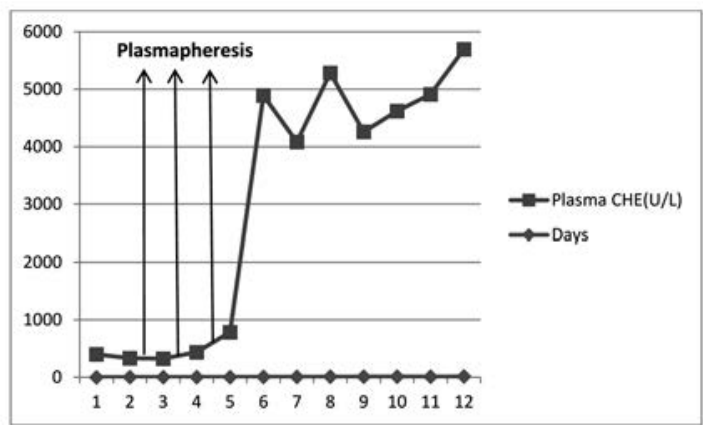

CHE: Cholinesterase

Fig. 2. The level of cholinesterase before and after plasmapheresis in Case 2.

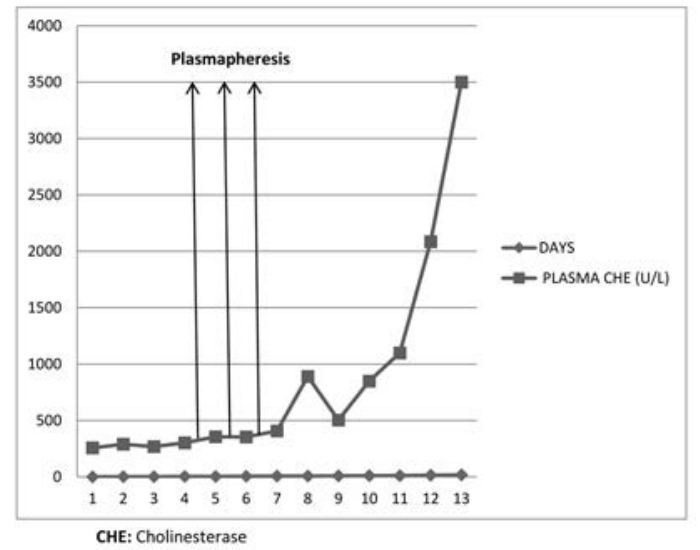

Fig. 3. The level of cholinesterase before and after plasmapheresis in Case 3

of Patient\# 1; was brought to our pediatric ES 3 days after her hair was washed with an agricultural pesticide containing $\mathrm{OP}$ to fight head lice with vomiting, abdominal pain and progressive deterioration of consciousness. At the admittance, her body temperature was $36.5^{\circ} \mathrm{C}$, respiratory rate was $28 / \mathrm{min}$, blood pressure was $110 / 60 \mathrm{mmHg}$, heart rate was $156 / \mathrm{min}$, and SpO2 was \%97. Her general condition was poor. She was intubated, with miotic and pinpoint pupils, rough lung sounds, widespread crepitane rales, just as her sister. She had thick secretion in oropharynx with GCS $11 / 15$. The level of plasma pseudocholinesterase was $398 \mathrm{U} / \mathrm{L}$. Monitoring clinical signs, she was given atropine at the dose of $0.05 \mathrm{mg} / \mathrm{kg} /$ hour every 5 minutes. Since heavy bronchorrhea and secretions continued despite intermittent atropine infusion, atropine infusion dose was augmented to $0.08 \mathrm{mg} / \mathrm{kg} /$ hour and injection of PAM at $40 \mathrm{mg} / \mathrm{kg} /$ dose was applied, followed by PAM infusion ( $8 \mathrm{mg} / \mathrm{kg} /$ hour) for two days. In spite of sixty hours of intensive therapy, the clinical findings did not improve and plasmapheresis was planned. The patient received plasmapheresis in three consecutive days. Consequently, the clinical signs improved, and the pseudocholinesterase level reached up to $4,073 \mathrm{U} / \mathrm{L}$ (Fig. 2). The patient was separated from mechanical ventilation and she gained consciousness. Atropine was decreased gradually and terminated on the twelfth day. The patient was discharged on the sixteenth day, similarly with her sister.

\section{Case 3}

A 14-year-old male patient admitted to our ES with vomiting, abdominal pain and progressive decline of consciousness after taking an agricultural pesticide (Dimethoate) for committing suicide. At the admittance his body temperature was $36.7^{\circ} \mathrm{C}$, blood pressure was $127 / 76 \mathrm{mmHg}$, heart rate was $68 / \mathrm{min}$ and, $\mathrm{SpO} 2$ was $95 \%$. His general condition was poor. He was intubated, with miotic and pinpoint pupils, rough lung sounds and widespread crepitane rales. GCS was found $10 / 15$. Pseudocholinesterase level was found $257 \mathrm{u} / \mathrm{L}$. Monitoring clinical signs, she was given atropine at the dose of $0.05 \mathrm{mg} / \mathrm{kg} /$ hour every 5 minutes. Since heavy bronchorrhea and secretions continued despite intermittent atropine infusion, atropine infusion dose was augmented to $0.08 \mathrm{mg} / \mathrm{kg} /$ hour and injection of PAM $40 \mathrm{mg} / \mathrm{kg} /$ dose was applied, followed by PAM infusion ( $8 \mathrm{mg} / \mathrm{kg} /$ hour) for two days. Plasmapheresis was applied for 3 days when the patient did not clinically improve after 96 hours of medical treatment. Atropine infusion and 
intermittent atropine injections were pursued. The patient gained consciousness and on the tenth day of hospitalization atropine injections were applied every 20 minutes. The patient was separated from mechanical ventilation and he gained consciousness. Atropine was decreased gradually and terminated on the sixteenth day. The patient totally recovered with pseudocholinesterase level of 3,630 U/L (Fig. 3). Patient was discharged on the eighteenth day.

Written informed consents were obtained from patients' parents who were mentioned in this paper.

\section{Discussion}

Our case reports show that plasmapheresis may be an option for OP poisoning when medical treatment is insufficient. All three pediatric patients who were exposed to OP totally recovered with plasmapheresis application following failure of medical treatment with atropine and oximes.

Because of the high morbidity and mortality risk of exposure, OP is a major health problem in developing countries. ${ }^{1}$ OP compounds are attached to the enzyme AchE and lead to the inactivation of the serine hydroxyl group of the enzyme by phosphorylating it. The reactivation of the phosphorylated enzyme occurs spontaneously (deacetylation, dephosphorylation), hydrolytic and by aging process. ${ }^{8}$ Aging is a time dependent process and expresses the loss of reactivation ability of the nucleophilic agents. It occurs by the activation of AChE or BuChe (butyryl cholinesterase) by dealkylation of the phosphoryl group following the phosphorylation of serin. ${ }^{8}$

The inhibition of $\mathrm{AChE}$ in $\mathrm{OP}$ poisoning leads $\mathrm{ACh}$ to cause stimulation in nicotinic receptors (autonomic ganglions and motor end plates) and muscarinic receptors (hearth, smooth muscles, glands). Increasing amount of ACh triggers off the clinical effects on the cholinergic synapses such as central nervous system, neuromuscular junction and autonomic ganglion. The first cholinergic crisis requires immediate treatment and the patient needs to be admitted into the in intensive care unit.9,10

Conventional therapy for OP poisoning includes supportive therapy and antidotal treatment. Atropine (anticholinergics) and oximes (cholinesterase reactivator) such as pralidoxime and obidoxime are the main medications used for antidotal treatment. Atropine eliminates parasympathetic stimuli by competitively blocking the effect of ACh on muscarinic receptors. Oximes, help in reversing the nicotinic effects which atropine cannot fix and in restoring active cholinesterase while reactivating AChE by dealkylating; removing the phosphoryl group on $\mathrm{AChE}$ which the OP binds. ${ }^{4}$ In some cases, however, these treatments are not sufficient to prevent morbidity and mortality. ${ }^{5}$ In a case series study carried out for the treatment of OP poisoning, the atropine and pralidoxime treatment did not have better results when compared to the atropine only treatment. ${ }^{7}$ In a randomized controlled trial, in which patients treated with low-dose and high-dose oximes were compared, it has been observed that highdose oxime treatment increased mortality. ${ }^{10}$ The reasons for the shortage of oxime therapy are as follows: 1- inadequate doses, 2- long half-life of OP compared to oximes with short half-life, 3- timing of the oxime therapy (late start, early finish). Besides, the people with BuChe D70G mutation may also be resistant to the reactivation of the enzyme. In addition, cholinesterase inhibition and hepatotoxicity can be seen as a result of oxime therapy. Because of these reasons, new strategies should be developed for OP poisoning. ${ }^{11}$

Serum cholinesterase activity is a good indicator for evaluating the clinical improvement of a patient. Balali-Mood et al. ${ }^{12}$ showed that there was a correlation between the clinical improvement of the patients with OP poisoning and the reactivation of the cholinesterase. If there is no gradual increase in the serum cholinesterase levels, prognosis might be poor with these patients. Cholinesterase reactivators are frequently used in patients with adequate cholinesterase levels, but these agents may be insufficient for some patients. Therefore, it is aimed to increase cholinesterase levels by using blood products. Fresh-frozen plasma contains many plasma proteins including cholinesterase. ${ }^{13}$ For some patients with low levels of cholinesterase, fresh frozen plasma is used for replacement. ${ }^{14}$ In preventing morbidity and mortality, atropine and / or PAM therapy added to the plasma treatment 
has been found more efficient than sole atropine and/or PAM therapy. ${ }^{15}$ Guven et al. ${ }^{16}$ recently reported an adult patient with OP poisoning who did not respond to atropine and PAM treatment; however, the clinical signs and cholinesterase levels increased after plasmapheresis. Depending on the absence of sepsis, increase in cholinesterase levels after plasmapheresis, improvement in clinical signs and decrease in atropine need, it would be reasonable to tell that our patients had benefited from plasmapheresis.

Plasmapheresis is a nonselective method which is used to dispose hazardous and toxic products from the body circulation. Plasmapheresis provides active cholinesterase by rapidly removing undesired inactive plasma cholinesterase. ${ }^{17}$ Two different techniques are used in plasmapheresis treatment: continuous filtration technique (Prisma) and centrifuge technique (Spectra-optica). Spectra- optica system uses tube set and a PL-1 kit in the double needle set. ${ }^{18}$ We preferred Spectraoptica system through a double-lumen central catheter, using citrate and heparin for anticoagulation for the plasmapheresis treatment in three consecutive days in patient 1 and patient 2 . In patient 3 , we used continuous filtration technique (Prisma) and maintained anticoagulation with heparin (Table I).

Yesilbas et al. ${ }^{17}$ reported a pediatric patient with OP poisoning who did not respond to atropine and PAM treatment; however, the clinical signs and cholinesterase levels increased after plasmapheresis, high-volume hemodiafiltration, and lipid infusion. They showed that AChE levels increased after this treatment. In a meta-analysis performed in China, six trials including 433 patients were identified. Treatment group including 211 patients adopted traditional physician therapy (atropine and PAM treatment) plus plasma exchange, and control group including 222 patients received physician therapy only. Mortality rates of OP poisoning patients treated with plasmapheresis were found to be lower when compared to the control group ${ }^{19}$.

In conclusion, plasmapheresis can be used in OP poisoning for patients who do not respond to the conventional treatment. However, further randomized controlled studies are necessary to have a clearer idea on this issue.

\section{REFERENCES}

1. Eddleston M. Patterns and problems of deliberate self-poisoning in the developing world. QJM 2002; 93: 715-731.

2. Kwong TC. Organophosphate pesticides: Biochemistry and clinical toxicology. Ther Drug Monit 2002; 24: 144-149.

3. Eddleston M, Szinicz L, Eyer P, et al. Oximes in acute organophosphorus pesticide poisoning: A systematic review of clinical trials. QJM 2002; 95: 275-283.

4. Sivagnanam S. Potential therapeutic agents in the management of organophosphorus poisoning. Crit Care 2002; 6: 260-261.

5. Johnson MK, Jacobsen D, Meredith TJ, et al. Evaluation of antidotes for poisoning by organophosphorus pesticides. Emerg Med 2000; 12: 22-37.

6. Naik B, Hirshhorn S, Dharnidharka VR. Prolonged neuromuscular block due to cholinesterase depletion by plasmapheresis. J Clin Anesth 2002; 14: 381-384.

7. Busund R, Koukline V, Utrobin U, et al. Plasmapheresis in severe sepsis and septic shock: A prospective, randomised, controlled trial. Intensive Care Med 2002; 28: 1434-1439.

8. Worek F, Diepold C, Eyer P. Dimethylphosphorylinhibited human cholinesterases: inhibition, reactivation, and aging kinetics. Arch Toxicol 1999; 73: 7-14.

9. De Bleecker JL. The intermediate syndrome in organophosphate poisoning: an overview of experimental and clinical observations. J Toxicol Clin Toxicol 1995; 33: 683-686.

10. Johnson S, Peter JV, Thomas K, et al. Evaluation of two treatment regimens of pralidoxime (1 gm single bolus dose vs. $12 \mathrm{gm}$ infusion) in the management of organophosphorus poisoning. J Assoc Phys India 1996; 44: 529-531.

11. Masson P, Froment MT, Bartels CF, Lockridge O. Importance of aspartate-70 in organophosphate inhibition, oxime re-activation and aging of human butyrylcholinesterase. Biochem J 1997; 325: 53-61.

12. Balali-Mood M, Shariat M. Treatment of organophosphate poisoning. Experience of nerve agents and acute pesticide poisoning on the effects of oximes. J Physiol Paris 1998; 92: 375-378.

13. Pichamuthu K, Jerobin J, Nair A, et al. Bioscavenger therapy for organophosphate poisoning - an openlabeled pilot randomized trial comparing fresh frozen plasma or albumin with saline in acute organophosphate poisoning in humans. Clin Toxicol 2010; 48: 813-819.

14. Smith DC, Ridley SA, Donaldson KF. Fresh frozen plasma and edrophonium in a patient with a plasma cholinesterase deficiency. Anaesthesia 1993; 48: 511513.

15. Dayananda VP, Bhaskara B, Pateel G. A study of effectiveness of fresh frozen plasma in organophosphorous compound poisoning in reducing length of Intensive Care Unit stay and in reducing need for tracheostomy. Anesth Essays Res 2016; 10: 268-272. 
16. Guven M, Sungur M, Eser B. The effect of plasmapheresis on plasma cholinesterase levels in a patient with organophosphate poisoning. Hum Exp Toxicol 2004; 23: 365-368.

17. Yesilbas O, Kihtir HS, Altiti M, et al. Acute severe organophosphate poisoning in a child who was successfully treated with therapeutic plasma exchange, high-volume hemodiafiltration, and lipid infusion. J Clin Apher 2016; 31: 467-469.
18. Demirkol D, Yildizdas D, Bayrakci B, et al Hyperferritinemia in the critically ill child with secondary HLH/sepsis/MODS/MAS: What is the treatment? Crit Care 2012; 16: R52.

19. Qiu HM, Zheng SC, Wan WG, et al. Meta-analysis of the effectiveness of plasma exchange in treatment of severe and acute organophosphate poisoning. Zhonghua Lao Dong Wei Sheng Zhi Ye Bing Za Zhi 2011; 29: 779-781. 